CrossMark

\&cick for updates

Cite this: Soft Matter, 2014, 10, 7096

\title{
Gas dissolution in antibubble dynamics
}

\author{
B. Scheid, ${ }^{\star a}$ J. Zawala ${ }^{c}$ and S. Dorbolo
}

Antibubbles are ephemeral objects. Their lifetime is driven by the slow drainage of the air shell from the bottom to the top of the antibubble under the action of hydrostatic pressure. We show in this paper that this argument is only valid if the water used to make the surfactant mixture is saturated in air. Otherwise, two paths are used by the air, which conduct to the thinning and the eventual collapse of the air shell: the drainage from the bottom to the top of the antibubble and the dissolution of the air into the liquid. Using degassed water dramatically shortens the lifetime of the antibubbles, as observed experimentally and rationalised by time-dependent simulations. Consequently, the antibubble lifetime is not only correlated with physical and chemical properties of the air-liquid interface but also with the gas content of the liquid. We also show that pure gas dissolution does not depend on the antibubble radius, a behaviour that allows to rationalise unexplained experimental data found in literature.

Received 2nd April 2014

Accepted 3rd June 2014

DOI: $10.1039 / c 4 s m 00718 b$

www.rsc.org/softmatter the air shell and the liquid bulk has remained an open question that we investigate in this work.

The permeability of an air-liquid interface is a crucial problem regarding gas exchanges $\left(\mathrm{O}_{2}, \mathrm{CO}_{2}, \ldots\right)$. As suggested by Caskey and Barlage ${ }^{10}$ and verified experimentally by Hanwright et al. ${ }^{11}$ for DTAB, water-soluble surfactants do not lower the permeability of the gas-liquid interface, contrarily to most of the insoluble surfactants. This was found to be independent of the surfactant concentration. Therefore, even full monolayer coverage of the soluble surfactant does not restrict the interfacial mass transfer of gas molecules, as compared to a clean interface.

In the core of the antibubble, the fluid is at rest soon after its formation and the inner liquid-air interface is quiescent, in the rheological properties of the liquid-air interface ${ }^{3}$ For surfactants with fast adsorption kinetics as compared to the drainage dynamics, we showed that the larger the surface shear viscosity, the longer the lifetime. ${ }^{4}$

Surface rheology has also been found to be important for the stability of foams, ${ }^{5,6}$ for which the surfactant nature and concentration are determinant as they influence both the formation of foam films and their drainage, ${ }^{7,8}$ leading in some conditions to common and Newton black films. ${ }^{9}$

In foams, the liquid fraction is usually very small and the gas dissolution is never a factor influencing the stability, while it should be the opposite for antibubbles that have a very small gas fraction. Yet the loss of air through the interface between

${ }^{a}$ TIPs, Fluid Physics Unit, Université Libre de Bruxelles, B-1050 Bruxelles, Belgium. E-mail: bscheid@ulb.ac.be

${ }^{b}$ Jerzy Haber Institute of Catalysis and Surface Chemistry Polish Academy of Sciences, Niezapominajek 8, 30-239 Krakow, Poland

${ }^{c}$ GRASP, Département de Physique B5, Université de Liège, B-4000 Liège, Belgium
Fig. 1 Schematic profile of an antibubble. The red arrows symbolise the hydrostatic drainage of the air from the bottom to the top of the antibubble and the green arrows the dissolution of the air by the liquid. 
case of which the mass transfer is governed by molecular diffusion, as in the experiments by Hanwright et al. This is not specifically true for the outer interface when the antibubble rises due to its density, slightly lower than that of the surrounding liquid. The flow then wipes out the diffusion boundary layer near the interface, which drastically increases the rate of mass transfer. Several techniques can be found in the literature to measure the convective mass transfer coefficient with moving interfaces: radioactive method, ${ }^{12}$ liquid laminar jet absorption, ${ }^{10}$ and bubbling method. ${ }^{13-15}$ When surfactant mixtures are concerned, some relevant trends are observed. First, the presence of surfactant molecules at the interface is found to decrease the mass transfer coefficient by essentially modifying the slip velocity and hence the flow structure in the vicinity of the interface. This was shown for cationic and anionic surfactants $\left(\mathrm{C}_{12} \mathrm{H}_{25} \mathrm{~N}\left(\mathrm{CH}_{3}\right)_{3} \mathrm{Cl}, \mathrm{C}_{16} \mathrm{H}_{33} \mathrm{~N}\left(\mathrm{CH}_{3}\right)_{3} \mathrm{Cl}\right.$, and sodium dodecyl sulfate SDS), ${ }^{10}$ for DTABr ${ }^{16}$ and for Tween $80 .{ }^{17}$ In general, the decrease of the mass transfer coefficient between clean water and a mixture with a concentration of surfactant beyond the critical micelle concentration $(\mathrm{cmc}$ ) is found to be between 40 and $70 \%$. Second, the mass transfer coefficient increases with the temperature. ${ }^{18}$ This result was obtained in the case of the liquid film. Third, the longer the carbon chain of the surfactant, the lower the mass transfer coefficient. ${ }^{18}$

Now the rising velocity of an antibubble, typically of $1 \mathrm{~cm}$ $\mathrm{s}^{-1}$, is about 10 times slower than the one of a bubble such that the effect of surfactant on the convective mass transfer coefficient is expected to be much less significant for an antibubble than for a bubble. Furthermore, it has been shown that loading the core of an antibubble with salt allows tuning its density such that it may remain static inside the liquid bath. ${ }^{1}$ The antibubble is thus an interesting system in such a respect as it may possess two quiescent interfaces with a small volume of trapped air but a very large contact area, $a=2\left(4 \pi R^{2}\right)$, where $R$ is the antibubble radius, of about 5-10 $\mathrm{mm}$. The initial thickness $h_{0}$ of the air shell ranges between 1 and $5 \mu \mathrm{m}$ as measured by different methods. ${ }^{\mathbf{1 1 9 , 2 0}}$ The antibubble thus has a volume of trapped air, $V_{0}=4 \pi R^{2} h_{0}$, that corresponds to the volume of a bubble of about $1 \mathrm{~mm}$ of radius for a contact area that is two orders of magnitude larger than the air bubble.

In this paper, we present results concerning the lifetime of static antibubbles when the gas content of the liquid is varied. We show that the lifetime is dramatically decreased when the liquid has been degassed before the antibubble creation. Combining the Epstein-Plesset solution for non-steady gas dissolution through a spherical interface, ${ }^{21}$ with the drainage model we have previously proposed for air drainage between viscous interfaces, ${ }^{4}$ allow to rationalise our experimental results. Moreover, previous results that showed no dependence of the antibubble lifetime with its radius ${ }^{\mathbf{1 , 2 2}}$ are now explained.

\section{Experimental}

As the influence of the gas concentration is concerned, bidistilled water was prepared in two different ways before adding the surfactant. The degassed sample was made of freshly bi-distilled water that has been boiled for 15 minutes before
12 hours of cooling down in a sealed bottle. The saturated sample was made from the degassed sample into which air was bubbled during 3 hours.

Antibubbles were made using either the saturated or the degassed sample with Triton X-100 concentrated at 10 times the cmc $(=0.24 \mathrm{mM})$. The antibubbles are obtained by gently pouring the mixture into a fulfilled tank of the same mixture and at the same electrical potential. By adjusting the incoming flow, the antibubble is formed. ${ }^{23}$ The lifetime was then measured using a timer. In so doing, it is possible to establish cumulative distribution of the lifetimes and to compare these distributions for the different considered samples.

In Fig. 2, the cumulative distribution functions (CDF) of the antibubble lifetimes are presented. The different symbols refer to different concentrations of gas in the water used to make antibubbles. It is assumed that the increase of gas content in the liquid during the time of the experiment is negligible as compared to the amount of dissolved gas present at the beginning of the experiment, such that the gas concentration into the liquid remains constant. This assumption is verified further.

The CDF for the saturated sample (bubbling) is represented with red bullets. The mean lifetime is found to be $72 \mathrm{~s}$. The CDF for the degassed sample (boiled) is represented with blue squares. The mean lifetime in this case is very short, namely $2.5 \mathrm{~s}$, and it was very difficult to even produce antibubbles. The degassed sample was then kept in air for $24 \mathrm{~h}$ and the experiment was performed again. The results are presented with green diamonds in Fig. 2. The mean lifetime is then $22 \mathrm{~s}$. Finally, we waited another $24 \mathrm{~h}$ and repeated the experiment, whose results are plotted with black triangles in Fig. 2. The mean lifetime is $39 \mathrm{~s}$.

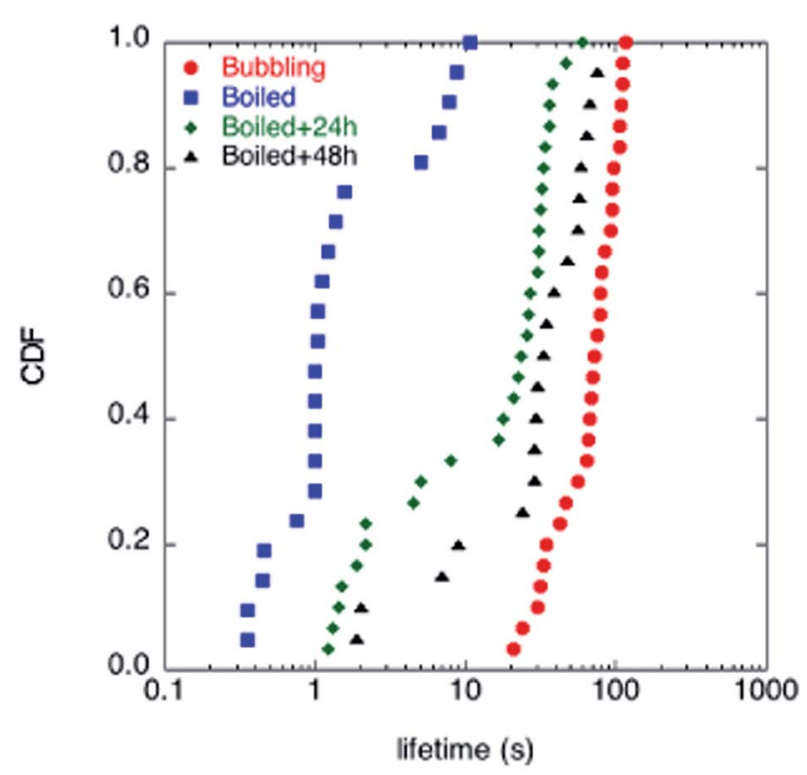

Fig. 2 Cumulative distribution functions of the lifetime of the antibubbles for four different mixtures made of water and Triton X-100 at 10 times the $\mathrm{cmc}$. The difference resides in the air content of the water used to produce the antibubbles. 
Even though we had no direct measurement of the concentration of the dissolved air for the intermediate samples (boiled $+24 \mathrm{~h}$ and boiled $+48 \mathrm{~h}$ ), the experimental data clearly demonstrate the important role of dissolution in the antibubble lifetime as it increases with the bulk concentration. Therefore, for the degassed sample, one can expect the lifetime to be largely influenced by dissolution, while for the saturated sample, the lifetime should only be governed by drainage. We have previously studied this later case ${ }^{4}$ and have shown the crucial role of surface rheology in the antibubble dynamics, leading to lifetimes of the order of $100 \mathrm{~s}$, similarly to the one obtained here for the saturated sample, even though with other surfactants. The much shorter lifetime obtained with the degassed sample indicates that dissolution dominates the drainage, the pure case of which is first analysed in the next section.

\section{Pure dissolution}

We assume here a perfectly static antibubble such that the mass transfer is limited by molecular diffusion. At constant temperature and pressure, the concentration of dissolved gas is assumed to be uniform and equal to $c_{\infty}$. We consider that the gas-liquid interfaces are at equilibrium, so that the surface concentration is at the dissolved gas concentration for a saturated solution, denoted by $c_{\mathrm{s}}$. Following Epstein and Plesset ${ }^{21}$ who studied the dissolution of a stationary bubble into a liquid, we write their time-dependent solution for the concentration gradient at the liquid-gas interface in spherical polar coordinate $r$, as follows:

$$
\left.\frac{\partial c}{\partial r}\right|_{R}=-\left(c_{\mathrm{s}}-c_{\infty}\right)\left(\frac{1}{R}+\frac{1}{\sqrt{\pi D t}}\right),
$$

where $c$ is the dissolved gas concentration in $\mathrm{kg} \mathrm{m}^{-3}$ and $D$ is the diffusion coefficient of the dissolved gas into the liquid. Additionally, the mass flow at both interfaces of the antibubble per unit time has the form

$$
\frac{\mathrm{d} m}{\mathrm{~d} t}=\left.2\left(4 \pi R^{2}\right) D \frac{\partial c}{\partial r}\right|_{R},
$$

and if $\rho_{\mathrm{a}}$ is the density of the air trapped into the antibubble, one also has

$$
\frac{\mathrm{d} m}{\mathrm{~d} t} \equiv \rho_{\mathrm{a}} \frac{\mathrm{d} V}{\mathrm{~d} t}=4 \pi R^{2} \rho_{\mathrm{a}} \frac{\mathrm{d} h}{\mathrm{~d} t},
$$

where $h(t)$ is the thickness of the air shell, which is assumed to be spatially uniform in the absence of drainage. Equating (3) and (2) with the use of (1) yields

$$
\frac{\mathrm{d} h}{\mathrm{~d} t}=-2 \frac{D}{R} S(1-\beta)\left\{1+\sqrt{\frac{\tau_{\mathrm{diff}}}{t}}\right\},
$$

where $S=c_{\mathrm{s}} / \rho_{\mathrm{a}}$ is the gas solubility into the liquid, $\tau_{\text {diff }}=$ $R^{2} /(\pi D)$ is the characteristic diffusion time, and $\beta=c_{\infty} / c_{\mathrm{s}}$ is the parameter that measures the degree of saturation: for $\beta=1$, the liquid is totally saturated in gas, such that the time for dissolution becomes infinite, whereas for $\beta=0$, the liquid is totally degassed and the time for dissolution is minimum. Integrating (4), with $h(0)=h_{0}$, yields

$$
h(t)=h_{0}-2 S \frac{D}{R}(1-\beta) t\left\{1+2 \sqrt{\frac{\tau_{\text {diff }}}{t}}\right\} .
$$

Taking $R=5 \mathrm{~mm}$ and $D=2 \times 10^{-9} \mathrm{~m}^{2} \mathrm{~s}^{-1}$ for dissolved air in water, ${ }^{3}$ one gets $\tau_{\text {diff }} \approx 4000 \mathrm{~s}$, which is orders of magnitude larger than the antibubble lifetime measured in experiments. One thus concludes that the dissolution process in antibubbles is always in the transient regime of the Epstein-Plesset solution,

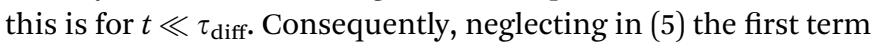
in the brackets, and searching for the complete dissolution time $t=\tau_{\mathrm{diss}}$, i.e. for $h\left(\tau_{\mathrm{diss}}\right)=0$, one obtains

$$
\tau_{\text {diss }}=\frac{\pi}{D}\left(\frac{h_{0}}{4 S(1-\beta)}\right)^{2} \text { for } \tau_{\text {diss }} \ll \tau_{\text {diff }} .
$$

Remarkably this expression does not depend on the antibubble radius, which corroborates previous experimental observations, ${ }^{1,22}$ a behaviour that has mainly remained unexplained until now.

The solubility of air in water can be considered to be an ideal, hence linear, combination of the solubility of the constituents of air in water. At atmospheric pressure and at $20^{\circ} \mathrm{C}$, using the data in ref. 24 and 25, we find $S=0.02$ (or $20 \mathrm{ml} \mathrm{l}^{-1}$ ). Considering the case of perfectly degassed liquid, i.e. for $\beta=0$, (6) becomes an exact expression for the lifetime, where $h_{0}$ is the only unknown parameter. Taking thus $\tau_{\text {diss }}=\hat{\tau}_{\text {life }}=2.5 \mathrm{~s}$ from our experiment, we find the initial film thickness, $h_{0}=3.2 \mu \mathrm{m}$, which enters the range of previously measured film thicknesses, between 1 and $5 \mu \mathrm{m} .{ }^{20}$ Of course, this value does not take into account the drainage inside the film, as discussed in the next section, such that the real initial thickness is probably a bit larger than $3.2 \mu \mathrm{m}$. Yet, keeping this value as a first guess for the initial film thickness, we report in Table 1 the values of the saturation parameter calculated by taking the dissolution time equals to the antibubble mean lifetime $\hat{\tau}_{\text {life }}$. We observe that after $24 \mathrm{~h}$ of the degassed solution in contact with air, the concentration is at $66 \%$ saturated. The volumetric concentration of dissolved gas is then merely $\beta S$, as indicated in Table 1. For the saturated solution (bubbling), the reason for obtaining $\beta$ $=0.81$ instead of 1 is because we have omitted the drainage here that also contributes to the thinning of the air shell and thus to the antibubble lifetime. The coupling between the drainage and dissolution is precisely the object of the next section. But two

Table 1 Saturation parameter $\beta$ obtained from (6) with $\tau_{\text {abs }}=\hat{\tau}_{\text {life, }}, h_{0}=$ $3.2 \mu \mathrm{m}, D=2 \times 10^{-9} \mathrm{~m}^{2} \mathrm{~s}^{-1}$ and $S=0.02$; s.d. states for the standard deviation of the experimental lifetimes

\begin{tabular}{llllc}
\hline & $\hat{\tau}_{\text {life }}(\mathrm{s})$ & s.d. $(\mathrm{s})$ & $\beta$ & $\beta S\left(\mathrm{ml} \mathrm{l}^{-1}\right)$ \\
\hline Boiled & 2.5 & 3.2 & 0 & 0 \\
Boiled + 24h & 22 & 16 & 0.66 & 13.2 \\
Boiled + 48 h & 39 & 25 & 0.75 & 15.0 \\
Bubbling & 72 & 29 & 0.81 & 16.2
\end{tabular}


assumptions have priority to be discussed, which are (i) constant bulk concentration and (ii) static conditions.

We assess first the assumption of constant bulk concentration during the time of the experiment, and especially the change in concentration in the confined core of the antibubble during its lifetime. Considering that, at maximum, half of the air volume, namely $2 \pi R^{2} h_{0}$, is dissolved into the core of the antibubble, it represents a volume ratio of $3 h_{0} /(2 R) \sim 10^{-3}$ or about $1 \mathrm{ml} \mathrm{l}^{-1}$, which is 20 times smaller than the solubility and should thus not significantly affect the air concentration in the liquid core, at least if it was fully degassed, as it would correspond to $\beta=0.05$ instead of 0 . In contrast, if nearly saturated, the core could reach the saturation during the lifetime of the antibubble, but then the rest of the air can still be transferred to the outer liquid volume - yet through a surface twice smaller which is not confined and thus big enough to dissolve the excess of air without changing its concentration.

Second, the short lifetimes observed for all samples could be explained by the residual motion of the antibubble, in the case of which the quiescent interface assumption does not hold and the mass transfer coefficient, as explained in the introduction, must be higher than the one in the pure diffusive case, i.e. $k_{1}=$ $D / R$. Nevertheless, the good order of magnitude found here for the dissolution time as compared to the 3 orders of magnitude difference existing between diffusive and convective mass transfer coefficients indicates that the antibubble residual motion does not play a significant role here. Moreover, one can reasonably assume that micrometric dust trapped in the film might also provoke the early rupture of some antibubbles.

\section{Modelling}

We use in this section the lubrication model developed in ref. 4 . Besides the asymptotic expansion based on the smallness of the aspect ratio $h_{0} / R \ll 1$, azimuthal symmetry is also assumed. The model then describes the time $(t)$ evolution of the film thickness $h(\theta, t)$, the pressure $p(\theta, t)$ and the surface velocity $u_{\mathrm{s}}(\theta, t)$ along the polar coordinate $\theta$, which ranges from $\theta=0$ at the South pole of the antibubble to $\theta=\pi$ at the North pole. The model accounts for (i) the hydrostatic pressure difference in the liquid that drains the air from the South to the North poles, (ii) the capillary pressure gradient due to small deformations of the outer interface as the air accumulates at the North pole due to drainage, (iii) the surface shear viscosity stresses due to the presence of surfactants at both interfaces, and (iv) the disjoining pressure gradient due to van der Waals interactions, which destabilises the air film primarily at the vicinity of the South pole where the film is the thinnest. It is assumed that the adsorption time of the surfactant molecules at the interface is much smaller than the lifetime such that no Marangoni effect is present and the surfactant surface density remains constant. The normal and tangential stress conditions are identical to ref. 4. The main equations are reproduced in appendix A for the sake of completeness. The only equation that should be modified to account for gas dissolution is the conservation equation, with the use of the Epstein-Plesset solution:

$$
\frac{\partial h}{\partial t}=\underbrace{-\nabla \cdot(h \hat{u})}_{\text {drainage }} \underbrace{-2 \frac{D}{R} S(1-\beta)\left\{1+\sqrt{\left.\frac{\tau_{\text {diff }}}{t}\right\}}\right.}_{\text {dissolution }},
$$

where $\nabla$ is the divergence operator (to be written in spherical coordinates) and $\hat{u}(\theta, t)=u_{\mathrm{s}}-h^{2} \partial_{\theta} p /\left(12 \mu_{\text {air }} R\right)$ is the crossaveraged velocity field in the frame of the lubrication approximation, where it is assumed that the surface velocity is identical on both interfaces. Note the factor 2 in (7) states for the two interfaces. The three-equation model for $h, p$ and $u_{\mathrm{s}}$ was solved under symmetric boundary conditions at both poles, namely $\partial_{\theta} h=\partial_{\theta} p=u_{\mathrm{s}}=0$ at $\theta=\{0, \pi\}$.

Neglecting the drainage in (7) leads back to (4), precisely obtained in the case of pure dissolution. The role of this modelling part is thus to study the effect of both dissolution and drainage whose relative effect is essentially tuned by the saturation parameter $\beta$. For the subsequent calculations, and using the same notations as in ref. 4 , also redefined in appendix A, we have fixed the surface tension to the equilibrium value of Triton$\mathrm{X}-100$ at $10 \mathrm{cmc}$, namely $\gamma=30 \mathrm{mN} \mathrm{m}^{-1}$, the water density to $\rho$ $=1000 \mathrm{~kg} \mathrm{~m}^{-3}$, the gravity acceleration to $g=9.81 \mathrm{~m} \mathrm{~s}^{-2}$, the dynamic viscosity of air to $\mu_{\text {air }}=1.85 \times 10^{-5}$ Pa s, the Hamaker constant for a liquid/air/liquid system to $A^{\prime}=4 \times 10^{-20} \mathrm{~J}^{26}$ the surface shear viscosity to $\varepsilon=0.27 \mathrm{mPa} \mathrm{s} \mathrm{m}$, and the diffusion coefficient to $D=2 \times 10^{-9} \mathrm{~m}^{2} \mathrm{~s}^{-1}$. Finally, and unless specified otherwise, the antibubble radius has been fixed to $R=5 \mathrm{~mm}$ and the initial film thickness to $h_{0}=3.2 \mu \mathrm{m}$, as obtained in the pure dissolution case.

The simulation results are plotted in Fig. 3 for various $\beta$. It shows thickness profiles at time intervals regularly spaced between $t=0$ and $t=\tau_{\text {life. }}$. In the case of degassed liquid $(\beta=0)$, the film thins everywhere at approximately the same rate, even though some residual drainage makes the film at the South pole slightly thinner than at the North pole. In this simulation, most of the air has been dissolved in a lifetime $\tau_{\text {life }}=1.9 \mathrm{~s}$, and at a decreasing rate due to the transient nature of the mass transfer. The lifetime is found to be slightly lower than in the pure dissolution case (see Table 1) because of the drainage that contributes to the thinning of the film, essentially at the South pole, where it destabilises first. It has been found that for any value of $\beta<0.29$ (as illustrated in Fig. 3 for $\beta=0$ ), the film thins for all $\theta$ due to dominant gas dissolution, whereas for $\beta>0.29$ the film thickens in the vicinity of the North pole where the air accumulates due to drainage, at a rate that is faster than the dissolution rate. This later situation is illustrated in Fig. 3 for $\beta=0.4$, which shows the formation of an air pocket of about $2.5 \mu \mathrm{m}$ at the North pole, in a lifetime of $\tau_{\text {life }}=3.8 \mathrm{~s}$. Finally, in the case of saturated liquid $(\beta=1)$, only the drainage is present and leads to a prominent air pocket of about $35 \mu \mathrm{m}$ at the North pole. The lifetime in this case is much longer, $\tau_{\text {life }}=75 \mathrm{~s}$, and is only governed by rheological properties of the interface, as reported in ref. 4 . Because the surface shear viscosity of the surfactant mixture is not known and very difficult to measure actually due to the extremely low shear rates involved, about $0.01 \mathrm{~Hz}$, the value of the surface shear viscosity has been tuned to $\varepsilon=0.27 \mathrm{mPa} \mathrm{s} \mathrm{m}$ to match the experimental mean lifetime with the simulated lifetime in the case of a saturated mixture 

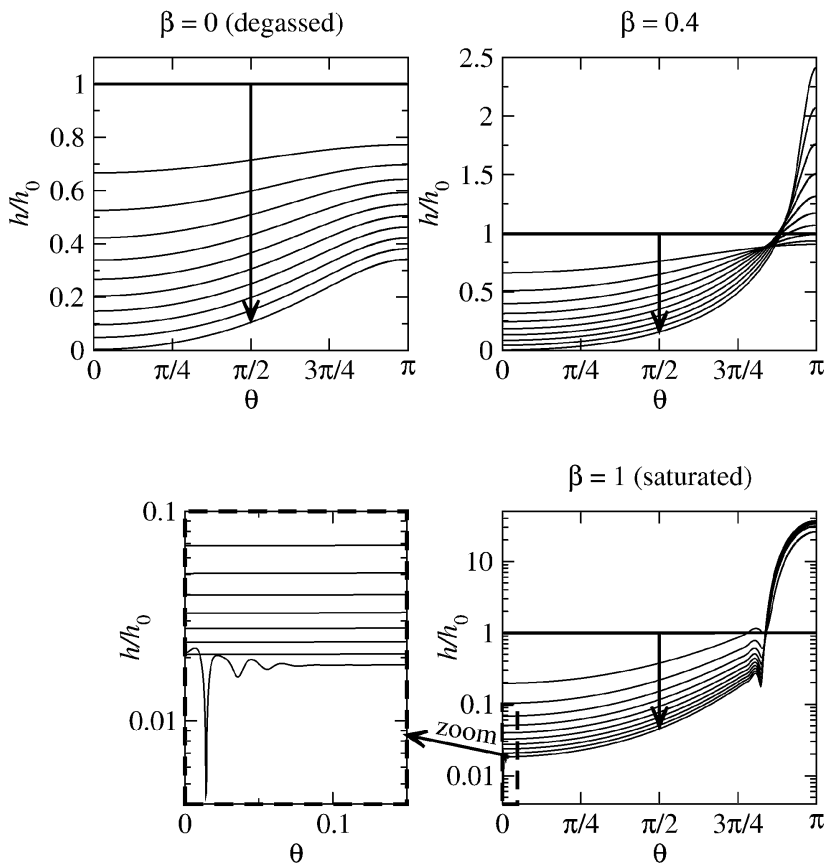

Fig. 3 Time evolution-as indicated by the vertical arrow-of the air film thickness profile $h(\theta, t)$ for three different values of the saturation parameter $\beta$, calculated for $R=5 \mathrm{~mm}$ and $h_{0}=3.2 \mu \mathrm{m}$. The time interval between the different profiles is $\tau_{\text {life }} / 10$, i.e. $0.19 \mathrm{~s}$ for $\beta=0$, $0.38 \mathrm{~s}$ for $\beta=0.4$ and $7.5 \mathrm{~s}$ for $\beta=1$. The initial solution is represented by the thick line. The logarithmic scale for $\beta=1$ allows to capture the prominent air pocket at the North pole and the film rupture event at the South pole, the zoom of which shows typical destabilisation process due to van der Waals interactions.

$(\beta=1)$. Increasing the surface shear viscosity to $\varepsilon=0.5 \mathrm{mPa} \mathrm{s} \mathrm{m}$ would increase the lifetime to $130 \mathrm{~s}$, corresponding approximately to the longest living antibubble observed in our experiments (see Fig. 2). However, such a fine tuning with the experimental data is abusive as the surface shear viscosity is not the only fitting parameter. Indeed, first, some dispersion exists in the radius $R$ measured on a large number of antibubbles; second, only an estimate for $h_{0}$ is available from the pure dissolution case, as obtained in the previous section; and last but not least, the saturation parameter $\beta$ is not known a priori, and even a small difference from unity can produce large variations in lifetime, as shown below.

As far as the antibubble collapse is concerned, which then determines the lifetime, a typical rupture event is shown in the zoomed image of Fig. 3 for $\beta=1$ : as the film thins and reaches a thickness of about $20 \mathrm{~nm}$, van der Waals interactions destabilise the film and break it on a time scale which is much smaller than the timescale for drainage. This is visualised in Fig. 4 by plotting the minimum film thickness as a function of time. The curve for $\beta=1$ clearly shows the nearly instantaneous break-up. The most significant effect comes now with the curve for $\beta=0.9$ that shows that if the bulk is only at $10 \%$ of the saturation, the lifetime drops by more than a factor 4 , to $17 \mathrm{~s}$. Similar curves arise for smaller values of the saturation parameter $\beta=0$ and 0.5 . For these curves, the dissolution rate becomes comparable to the growth rate of the van der Waals

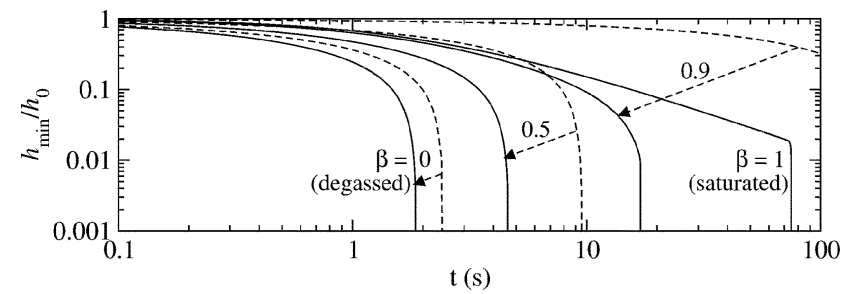

Fig. 4 Time evolution of the dimensionless minimum film thickness simulated for four values of the saturation parameter $\beta=0,0.5,0.9$, and 1 and calculated for $R=5 \mathrm{~mm}$ and $h_{0}=3.2 \mu \mathrm{m}$. The dashed lines are the solution (5) obtained in the case of pure dissolution and the dashed arrows then show the effect of drainage.

instability such that no separation of time scales between the drainage/dissolution dynamics and the rupture dynamics can be observed. Finally, the dashed lines in Fig. 4 are calculated using (5), which corresponds to the case of pure dissolution. As compared to the corresponding solid lines, which are related by the dashed arrows in Fig. 4, we can clearly assess the relative effects of drainage and dissolution on the antibubble dynamics: for $\beta=0$ the drainage shortens the lifetime by only $25 \%$, which makes the gas dissolution the governing mechanism; for $\beta=0.5$ the drainage shortens the lifetime by a factor 2 , in the case of which both effects are of the same importance; while for $\beta=0.9$, the drainage shortens the lifetime by a factor 12 as compared to pure dissolution, which makes the drainage the governing mechanism.

All our simulation results for $R=5 \mathrm{~mm}$ and $h_{0}=3.2 \mu \mathrm{m}$ are finally plotted in Fig. 5, where the solid line maps the antibubble lifetime with the saturation parameter. Similar results are also mapped in the dashed line for a larger antibubble of $R$ $=10 \mathrm{~mm}$ and in the dot-dashed line for a thicker initial film thickness of $h_{0}=5 \mu \mathrm{m}$. One sees that the three curves cover most of the range of lifetimes found experimentally in Fig. 2. In the region dominated by dissolution, namely for $\beta \rightarrow 0$, the antibubble lifetime essentially depends on the film thickness and not on the radius, as inferred from (6). The slight dependence on the radius is because of the drainage that also

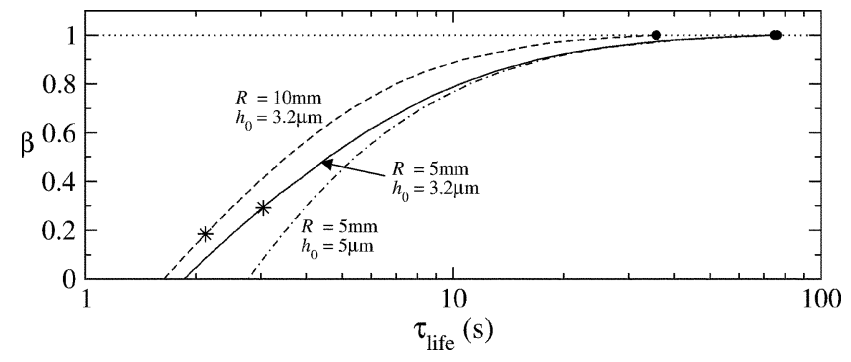

Fig. 5 Mapping between the simulated lifetime $\tau_{\text {life }}$ of an antibubble and the saturation parameter $\beta$, for three different antibubble dimensions. The largest lifetime is obtained for $\beta=1$ and is indicated with a filled circle for each curve. The stars indicate the parameter coordinates above which the film thickness starts to thicken above $h_{0}$ at the North pole due to the drainage. No star is shown on the dot-dashed line as the film always thickens at the North pole, even for $\beta=0$. 
contributes to the film thinning at the South pole and makes the lifetime smaller than the pure dissolution time (6). In contrast, in the region of pure drainage, namely for $\beta=1$, the smaller the antibubble radius, the longer the lifetime - compare the filled circles for each curve in Fig. 5 - since the time for drainage is inversely proportional to the driving hydrostatic pressure difference $2 \rho_{\mathrm{a}} R$. In this case the influence of the initial film thickness is negligible. Finally, Fig. 5 demonstrates the great sensitivity of the antibubble lifetime with the bulk concentration as it approaches saturation, i.e. as $\beta \rightarrow 1$. Given that none of the reported experimental data on antibubble lifetime $e^{\mathbf{1 , 2 , 2 0 , 2 2 , 2 3}}$ has ever mentioned the gas content of the surfactant mixtures, it appears difficult to discuss the dependence of the antibubble radius on the lifetime. Nevertheless, in at least two papers, ${ }^{\mathbf{1 , 2 2}}$ no such dependence was observed, which suggests that the role of gas dissolution in these experiments was significant.

\section{Conclusions}

The conclusion is that the lifetime of the antibubble is driven by the drainage of the air under the action of hydrostatic pressure plus the gas exchange through both interfaces of the air shell. The balance between both mechanisms depends on the degree of saturation of the liquid. Indeed, if the liquid has been degassed, the air contained in the shell rapidly dissolves in the bulk liquid, while if the liquid has been saturated, the air contained in the shell slowly drains at the North pole where it forms a bulge of amplitude of about 1 order of magnitude larger than the initial film thickness.

Modelling shows that the gas dissolution in antibubbles is always in the transient regime for diffusion as the lifetime of antibubbles is by at least one order of magnitude smaller than the time needed to build the concentration boundary layer in the liquid after the formation of the antibubble.

This work, coupling experiments and modelling, demonstrates that the air content of the liquid is a very important parameter concerning the antibubble. First, for comparing the rheological properties of the interface of two mixtures, the same water should be used. Secondly, it shows that the dependence of the lifetime with the antibubble radius is non-trivial as they are independent of pure dissolution and inversely proportional for pure drainage, the reality being most of the cases intermediate between these two extremes. Thirdly, the experiments show that the antibubble can be a simple and cheap mean to measure the air content of a liquid after establishing the relationship between the lifetime and the absolute air content of the liquid, as proposed in Fig. 5. Therefore our results finally suggest to use the antibubble as a basic sensor of the air content in a liquid, provided the thickness is estimated a priori in the case of a degassed mixture, hence using (6) with $\beta=0$.

\section{Appendix A}

We reproduce below the system of equations as numerically solved in this work. The first equation is the dimensionless form of the conservation equation given in (7), while the two last equations are the dimensionless normal and tangential stress boundary conditions, which are identical to eqn (2) and (3) in ref. 4:

$$
\begin{aligned}
& \frac{\partial \bar{h}}{\partial \bar{t}}=-\frac{1}{\sin (\pi \bar{\theta})} \frac{\partial}{\partial \bar{\theta}}\left[\bar{h} \sin (\pi \bar{\theta})\left(\bar{u}_{\mathrm{s}}-\frac{\bar{h}^{2}}{6} \frac{\partial \bar{p}}{\partial \bar{\theta}}\right)\right] \\
&-2 S t(1-\beta)\left(1+\frac{1}{\sqrt{t / \tau_{\text {trans }}}}\right), \\
& \frac{\partial \bar{p}}{\partial \bar{\theta}}=\frac{1}{2} \frac{\partial}{\partial \bar{\theta}}\left[\cos (\pi \bar{\theta})-\frac{\mathrm{Bo}}{\sin (\pi \bar{\theta})} \frac{\partial}{\partial \bar{\theta}}\left(\sin (\pi \bar{\theta}) \frac{\partial \bar{h}}{\partial \bar{\theta}}\right)+\frac{A}{\bar{h}^{3}}\right], \\
& \bar{h} \frac{\partial \bar{p}}{\partial \bar{\theta}}=2 \mathrm{~Bq}\left[\frac{\partial}{\partial \bar{\theta}}\left(\frac{1}{\sin (\pi \bar{\theta})} \frac{\partial}{\partial \bar{\theta}}\left(\sin (\pi \bar{\theta}) \bar{u}_{\mathrm{s}}\right)\right)+\pi^{2} \bar{u}_{\mathrm{s}}\right] .
\end{aligned}
$$

The bars indicate dimensionless quantities. The independent variables $\theta$ and $t$ have been scaled, respectively, with $\pi$ and $\tau_{0}=$ $\mu_{\text {air }} \pi^{2} R /\left(\rho_{\mathrm{a}} h_{0}{ }^{2}\right)$ corresponding to the lubrication timescale for drainage. The dependent variables $h, u_{\mathrm{s}}$ and $p$ have been scaled, respectively, with $h_{0}, \pi R / \tau_{0}$, and $p_{0}=2 \rho_{\mathrm{a}} R$, which represents the hydrostatic pressure difference driving the air shell drainage. The dimensionless numbers are defined as follows:

$$
\begin{gathered}
\mathrm{St}=\frac{h_{0} R}{\tau_{0} D S}, \quad \text { Bo }=\frac{2 \gamma h_{0}}{p_{0} \pi^{2} R^{2}}, \\
A=\frac{A^{\prime}}{3 \pi p_{0} h_{0}^{3}}, \quad \mathrm{~Bq}=\frac{\varepsilon h_{0}}{\mu_{\text {air }} \pi^{2} R^{2}},
\end{gathered}
$$

in which the parameters are defined in the core of the paper. The Stanton number (St) compares the air dissolution rate to the rate of drainage, the Bond number (Bo) compares the capillary pressure to the hydrostatic pressure, the Hamaker number $(A)$ compares the disjoining pressure to the hydrostatic pressure, and the Boussinesq number (Bq) compares the surface shear viscous stress to the air viscous stress.

\section{Acknowledgements}

We thank the two anonymous referees for their crucial suggestions of considering the Epstein-Plesset solution, which has fundamentally changed the numerical results of the paper. We gratefully thank Jan Vermant and Joe Samaniuk for surface property measurements of surfactant mixtures. S. D. and B. S. thank the Fonds de la Recherche Scientifique - FNRS for financial support. J. Z. thanks the PAN/FNRS research agreement (2010-2013) for financial support. This research was funded by the Inter-university Attraction Pole Programme (IAP 7/38 MicroMAST) initiated by the Belgian Science Policy Office. This work was performed under the umbrella of the COST action MP1106.

\section{References}

1 S. Dorbolo, E. Reyssat, N. Vandewalle and D. Quéré, Europhys. Lett., 2005, 69, 966.

2 S. Dorbolo, D. Terwagne, R. Delhalle, J. Dujardin, N. Huet, N. Vandewalle and N. Denkov, Colloids Surf., A, 2010, 365, 43. 
3 E. L. Cussler, Diffusion: Mass Transfer in Fluid Systems, Cambridge University Press, New York, 1997.

4 B. Scheid, S. Dorbolo, L. R. Arriaga and E. Rio, Phys. Rev. Lett., 2012, 109, 264502.

5 M. Durand and H. A. Stone, Phys. Rev. Lett., 2006, 97, 226101.

6 A. T. Poortinga, Langmuir, 2011, 27, 2138.

7 S. I. Karakashev and D. S. Ivanova, J. Colloid Interface Sci., 2010, 343(2), 582.

8 K. I. Khristov, D. R. Exerowa and P. M. Kruglyakov, Colloid Polym. Sci., 1983, 261, 265.

9 D. Exerowa, P. M. Kruglyakov, Foam and foam films - theory, experiment, application, Elsevier, Amsterdam, 1998.

10 J. A. Caskey and W. B. Barlage, J. Colloid Interface Sci., 1972, 41, 52 .

11 J. Hanwright, J. Zhou, G. M. Evans and K. P. Galvin, Langmuir, 2005, 21, 4912.

12 J. G. Hawke and A. G. Parts, J. Colloid Sci., 1964, 19, 448.

13 W. D. Deckwer, R. Burckhart and G. Zoll, Chem. Eng. Sci., 1974, 29, 2177.

14 J. M. T. Vasconcelos, J. M. L. Rodrigues, S. C. P. Orvalho, S. S. Alves, R. L. Mendes and A. Reis, Chem. Eng. Sci., 2003, 58, 1431.
15 G. Vázquez, M. A. Cancela, C. Riverol, E. Alvarez and J. M. Navaza, Chem. Eng. J., 2000, 78, 13.

16 D. Gómez-Díaz, J. M. Navaza and B. Sanjurjo, Ind. Eng. Chem. Res., 2009, 48, 2671.

17 I. Belo, A. García-Albuín, D. Gómez-Díaz, J. M. Navaza and I. Vidal-Tato, Chem. Eng. Technol., 2011, 34, 1790.

18 R. Farajzadeh, R. Krastef and P. L. J. Zitha, Adv. Colloid Interface Sci., 2008, 137, 27.

19 S. Dorbolo, N. Vandewalle, E. Reyssat and D. Quéré, Europhys. News, 2006, 37, 24.

20 W. Suhr, Eur. J. Phys., 2012, 33, 443.

21 P. S. Epstein and M. S. Plesset, J. Chem. Phys., 1950, 18, 1505. 22 P. G. Kim and J. Vogel, Colloids Surf., A, 2006, 289, 237.

23 P. G. Kim and H. A. Stone, Europhys. Lett., 2008, 83, 54001.

24 R. Battino, Oxygen and Ozone, IUPAC Solubility Data Series, Pergamon Press, Oxford, England, 1981, vol. 7.

25 Nitrogen and Air, IUPAC Solubility Data Series, ed. R. Battino, Pergamon Press, Oxford, England, 1982, vol. 10.

26 J. Israelachvili, Intermolecular and Surface Forces, Academic Press, Amsterdam, 3rd edn, 2011. 